\title{
A STABILITY THEOREM FOR A REAL ANALYTIC SINGULAR CAUCHY PROBLEM
}

\author{
W. J. WALKER
}

ABSTRACT. In this paper we prove the equation $u_{t t}-t^{2} u_{x x}-$ $a(t) u_{x}=0, p>0$, with initial conditions $u(x, 0)=\alpha(x), u_{t}(x, 0)=\beta(x)$ is well posed provided that $\alpha(x)$ and $\beta(x)$ belong to special classes of real analytic functions. In general this problem is not stable for $p>1$ and $\alpha(x)$ and $\beta(x)$ real analytic functions.

1. Introduction. Let $A(R)$ be the class of all real valued functions which are represented by power series expansions on the interval $(-R, R)$. In general the Cauchy problem

$$
\begin{gathered}
u_{t t}-t^{2 p} u_{x x}-a(t) u_{x}=0, \quad p>0, \\
u(x, 0)=\alpha(x), \quad u_{t}(x, 0)=\beta(x),
\end{gathered}
$$

is not well posed if $\alpha(x)$ and $\beta(x)$ belong to $A(R)$. In fact for $p>1$ the stability in the uniform metric may be violated (for an example see [1]).

In this paper we define special classes $H(R)$ of functions in $A(R)$ and prove a theorem giving a well-posed problem provided $\alpha(x)$ and $\beta(x)$ are restricted to belong to such a class $H(R)$. M. H. Protter [6] gave a condition for a more general problem which implies that (1), (2) is well posed if $\lim _{t \rightarrow 0+} t^{1-p} a(t)=0$. For further papers on problems of this nature see $([1],[3],[4],[5],[8])$. For general abstract existence and uniqueness theorems see [2] and [3]. In particular A. B. Nersesjan in [5] states a theorem which shows that the Cauchy problem is well posed in the case that $z$ is a complex variable, $\alpha(z)$ and $\beta(z)$ are analytic for $|z|<R$ and solutions are admitted in the class of functions $u(z, t)$ such that, for each fixed $t$, $u(z, t)$ is analytic for $|z|<R$. We shall see that stability occurs for $p>1$ in the complex variable case, as opposed to the real variable case, because of the availability of the Cauchy estimates. Namely if $z$ is complex and

$$
\max _{0 \leqq|z| \leqq \rho}\left|\sum_{n=0}^{\infty} \alpha_{n} z^{n}-\sum_{n=0}^{\infty} \phi_{n} z^{n}\right|=\delta
$$

Received by the editors February 13, 1973.

AMS (MOS) subject classifications (1970). Primary 35A20, 35M05.

(C) American Mathematical Society 1974 
then for each $n,\left|\alpha_{n}-\beta_{n}\right| \leqq \delta / \rho^{n}$. Of course such estimates are not available in the case that $x$ is a real variable; however, we define a more general estimate of this nature which we call a $G$ estimate and show that in the real analytic case we have stability provided that $\alpha(x)$ and $\beta(x)$ are restricted to a class of functions which have the $G$ estimate property.

2. Definitions and preliminaries. Let $I$ be an index set and let $H(R)=\left\{\alpha_{h}(x)\right\}_{h \in I}$ be a class of functions in $A(R)$.

Definition. $H(R)$ will be said to have the $G$ estimate property if the following condition is satisfied: Suppose $0<\rho<R$ and $\alpha(x)=\sum_{n=0}^{\infty} \alpha_{n} x^{n}$ and $\phi(x)=\sum_{n=0}^{\infty} \phi_{n} x^{n}$ are any two functions in $H(R)$. Let

$$
\delta(\rho)=\max _{0 \leqq|x| \leqq \rho}|\alpha(x)-\phi(x)| ;
$$

then for every nonnegative integer $n$,

$$
\left|\alpha_{n}-\phi_{n}\right| \leqq g(n, \rho) \delta(\rho) / \rho^{n}
$$

where $g(n, \rho) \leqq c(\rho) \prod_{i=0}^{N}(n+i), c(\rho)$ positive and $N$ a fixed positive integer.

EXAMPLES. (i) Suppose $\left\{b_{n}(h)\right\}$ is a sequence of nonnegative monotone increasing functions each defined for $h \geqq 0$ and satisfying $b_{n}(h) \leqq 1$. Define

$$
H(1)=\left\{\sum_{n=0}^{\infty}(-1)^{n} b_{n}(h) x^{n}\right\}_{h \in[0, \infty)},
$$

then $H(1)$ has the $G$ estimate property with $g(n, \rho)=1$. It is easy to show that if $0 \leqq h<k$ and $z$ is a complex variable then

$$
\left|\sum_{n=0}^{\infty}(-1)^{n}\left(b_{n}(k)-b_{n}(h)\right) z^{n}\right|
$$

assumes its maximum on $|z|=\rho$ at $z=-\rho$. Then the Cauchy estimates for the disc $|z| \leqq \rho$ in the complex plane give the required results for the interval $|x| \leqq \rho$ on the real line.

(ii) A simple example in which $g(n, \rho)$ must be a function of $n$ is

$$
H(1)=\left\{h /\left(1+x^{2}\right)^{2}\right\}_{h \in[0, \infty)} .
$$

(iii) If $H(1)=\{\sin h x\}_{h \in[0, c)}$ we have a class of functions which can never satisfy the $G$ estimate property.

Definition. Suppose $0<r<\rho<R, 0<\theta, 0<X$,

$$
\boldsymbol{X}+\theta^{p+1} /(p+1)=r
$$


and $S=\{(x, t): 0 \leqq t \leqq \theta,|x| \leqq X\}$. The Cauchy problem (1), (2) is said to be $G$ stable on $S$ with respect to the class of functions $H(R)$ if for each $\varepsilon>0$ there exists $\delta>0$ such that whenever $\alpha_{1}(x), \alpha_{2}(x), \beta_{1}(x), \beta_{2}(x)$, belong to $H(R)$ and $\max _{0 \leqq|x| \leqq \rho}\left|\alpha_{1}(x)-\alpha_{2}(x)\right|<\delta, \max _{0 \leqq|x| \leqq \rho}\left|\beta_{1}(x)-\beta_{2}(x)\right|$ $<\delta$, then if $u_{i}(x, t)$ is the solution of (1), (2) with $\alpha(x)=\alpha_{i}(x), \beta(x)=\beta_{i}(x)$, $i=1,2$, it follows that

LEMMA.

$$
\max _{(x, t) \in S}\left|u_{1}(x, t)-u_{2}(x, t)\right|<\varepsilon .
$$

$$
\sum_{n=0}^{\infty} \sum_{v=0}^{\infty} \sum_{w=0}^{\infty} g(n+w+v) \frac{(n+w+v) !}{n ! w ! v !} \alpha^{n} \beta^{v} \frac{\gamma^{w}}{w !}
$$

converges for all $\gamma$ if $|\alpha|+|\beta|<1$.

Proof. Choose $\delta>0$ such that $|\alpha|+|\beta|+\delta<1$. Then there exists $w_{0}$ such that for all $w \geqq w_{0},|\gamma|^{w} / w !<\delta^{w}$. Now for each fixed $w<w_{0}$ the double series

$$
\sum_{n=0}^{\infty} \sum_{v=0}^{\infty} g(n+w+v) \frac{(n+w+v) !}{n ! v !} \alpha^{n} \beta^{v}
$$

converges. To prove this we observe that the Appell series

$$
\sum_{n=0}^{\infty} \sum_{v=0}^{\infty} \frac{(n+w+v) !}{n ! v !}\left(\alpha^{n} \beta^{v}\right)
$$

converges for $|\alpha|+|\beta|<1$ and the proof will be unaltered by the term $g(n+w+v)$ since $g(n) \leqq c(\rho) \prod_{i=0}^{N}(n+i)$ (for the proof of the convergence of the Appell series see [7, pp. 210-213]). For $w \geqq w_{0}$ the triple series is dominated by

$$
\sum_{n=0}^{\infty} \sum_{w=w_{0}}^{\infty} \sum_{v=0}^{\infty} g(n+w+v) \frac{(n+w+v) !}{n ! w ! v !}|\alpha|^{n}|\beta|^{n} \delta^{w}
$$

and again, as above, convergence follows since $|\alpha|+|\beta|+\delta<1$ (see Lauricella functions [7, p. 227]).

3. THEOREM. If the class of functions $H(R)$ has the $G$ estimate property then the Cauchy problem (1), (2) is $G$ stable on $S$ with respect to $H(R)$.

ProOF. Suppose $\alpha_{1}(x)-\alpha_{2}(x)=\sum_{n=0}^{\infty} \alpha_{n} x^{n}$,

$$
\beta_{1}(x)-\beta_{2}(x)=\sum_{n=0}^{\infty} \beta_{n} x^{n}, \quad 0<r<\rho<R,
$$

and $X+\theta^{p+1} /(p+1)=r$. We need only assume that $a(t)$ is continuous. 
Integrating (1) twice with respect to $t$, we obtain

$$
u-K u_{x x}-L u_{x}=\alpha(x)+t \beta(x)
$$

where the operators $K$ and $L$ are defined by

$$
(K f)(t)=\int_{0}^{t} \int_{0}^{r} s^{2 p} f(s) d s d r, \quad(L f)(t)=\int_{0}^{t} \int_{0}^{r} a(s) f(s) d s d r,
$$

for $f(t)$ a real valued continuous function defined on an interval $0 \leqq t \leqq \theta$.

We shall denote by $I(w, v)$ the summation over all distinct operators obtained by applying $L w$ times and $K v$ times and define $f_{n}(t)=\alpha_{n}+\beta_{n} t$. Let \| $\|$ denote the supremum norm on $C[0, \theta]$. Then by the $G$ estimate property

$$
\left\|f_{n}\right\| \leqq\left|\alpha_{n}\right|+\left|\beta_{n}\right| \theta \leqq(1+\theta) g(n) \delta / \rho^{n}
$$

where for convenience we use the abbreviations $g(n)=g(n, \rho)$ and $\delta=\delta(\rho)$. Substitution in (3) shows there will be a solution of the form $u(x, t)=$ $\sum_{n=0}^{\infty} a_{n}(t) x^{n}$ if, for each $n$,

$$
a_{n}(t)=(n+1)(n+2) K a_{n+2}(t)+(n+1) L a_{n+1}(t)+f_{n}(t) .
$$

Assuming for the moment that the infinite sum is absolutely convergent we will show that (4) is satisfied if, for all $n$,

$$
a_{n}(t)=\sum_{w=0}^{\infty} \sum_{v=0}^{\infty} \frac{(n+w+2 v) !}{n !} I(w, v) f_{n+w+2 v}(t)
$$

It can be seen that

$$
\text { for } w \geqq 1, v \geqq 1, I(w, v)=K I(w, v-1)+L I(w-1, v)
$$

for $w=0, v \geqq 1, I(0, v)=K I(0, v-1)$

for $w \geqq 1, v=0, I(w, 0)=L I(w-1,0)$.

Then it may be verified directly that (4) is an identity.

It remains to show the infinite sum converges absolutely and to prove $G$ stability on $S=\{(x, t),|x| \leqq X, 0 \leqq t \leqq \theta\}$.

We see that $I(w, v)$ consists of $\left(\begin{array}{c}w+v \\ v\end{array}\right)$ operators each obtained by applying $L w$ times and $K v$ times. Further, each operator has norm at most $\theta^{2 w+2 v(p+1)}\|a\| w /(2 w) ! E(v)$ where

$$
E(v)=\prod_{j=1}^{v}(2 p j+2 j-1)(2 p j+2 j) \geqq(p+1)^{2 v}(2 v) ! .
$$


Hence, if we set $T=\sum_{n=0}^{\infty}\left\|a_{n}\right\| X^{n}$ and denote $\sum_{n=0} \sum_{w=0} \sum_{v=0}$ by $\sum$,

$$
\begin{aligned}
T \leqq & \left(\begin{array}{c}
w+v \\
v
\end{array}\right) \frac{(n+w+2 v) ! X^{n} \theta^{2 w+2 v(p+1)}\|a\|^{w}(1+\theta) g(n+w+2 v) \delta}{n !(2 w) !(2 v) !(p+1)^{2 v} \rho^{n+w+2 v}} \\
\leqq & \left(\begin{array}{c}
w+v \\
v
\end{array}\right) \frac{(n+w+v) !}{n ! w ! v !}\left(\frac{X}{\rho}\right)^{n}\left(\frac{\theta^{(p+1)}}{(p+1) \rho}\right)^{v} \frac{1}{w !}\left(\frac{\theta^{2}\|a\|}{\rho}\right)^{w} \\
& \times(1+\theta) g(n+w+v) \delta .
\end{aligned}
$$

Now there exists $\gamma$ such that $0<\gamma<1$ and

$$
X / \rho+\left(\theta^{p+1} /(p+1) \rho\right)^{1-\gamma}<1
$$

and there exists $k>0$ such that

$$
\left(\theta^{p+1} /(p+1) \rho\right)^{\gamma}+k<1 .
$$

By the lemma and (5)

$$
\sum \frac{(n+w+v) !}{n ! w ! v !} g(n+w+v)\left(\frac{X}{\rho}\right)^{n}\left[\left(\theta^{p+1} /(p+1) \rho\right)^{1-\gamma}\right]^{v} \frac{\left(\theta^{2}\|a\| / \rho k\right)^{w}}{w !}
$$

converges. Hence there exists $M$ such that for all w and $v$

$$
\begin{aligned}
M k^{w} \geqq \sum_{n=0}^{\infty} & \frac{(n+w+v) !}{n ! w ! v !} g(n+w+v) \\
& \times\left(\frac{X}{\rho}\right)^{n}\left[\left(\theta^{\nu+1} /(p+1) \rho\right)^{1-\gamma}\right]^{v} \frac{\left(\theta^{2}\|a\| / \rho\right)^{w}}{w !} .
\end{aligned}
$$

Hence

$$
T \leqq M(1+\theta) \delta \sum_{w=0}^{\infty} \sum_{v=0}^{\infty}\left(\begin{array}{c}
w+v \\
v
\end{array}\right)\left[\left(\theta^{p+1} /(p+1) \rho\right)^{y}\right]^{v} k^{w} .
$$

The latter series converges by (6) and the proof is complete.

\section{REFERENCES}

1. I. S. Berezin, On Cauchy's problem for linear equations of the second order with initial conditions on a parabolic line, Mat. Sb. 24 (66) (1949), 301-320; English transl., Amer. Math. Soc. Transl. (1) 4 (1962), 415-439. MR 11, 112.

2. R. Carroll, Some degenerate Cauchy problems with operator coefficients, Pacific J. Math. 13 (1963), 471-485. MR 29 \#367.

3. R. Carroll and C. Wang, On the degenerate Cauchy problem, Canad. J. Math. 17 (1965), 245-256. MR 36 \#489.

4. Chi Min-you, The Cauchy problem for a class of hyperbolic equations with data on a line of parabolic degeneracy, Acta. Math. Sinica 8 (1958), 521-529=Chinese Math. 9 (1967), 246-254. MR 21 \#5815. 
5. A. B. Nersesjan, The Cauchy problem for degenerating hyperholic equations of second order, Dokl. Akad. Nauk SSSR 166 (1966), 1288-1291=Soviet Math. Dokl. 7 (1966), 278-281. MR 33 \#4465.

6. M. H. Protter, The Cauchy problem for a hyperbolic second order equation with data on the parabolic line, Canad. J. Math. 6 (1954), 542-553. MR 16, 255.

7. L. J. Slater, Generalized hypergeometric functions, Cambridge Univ. Press, Cambridge, 1966. MR 34 \#1570.

8. S. A. Tersenov, A prohlem with data given on a line of degeneracy for a system of hyperbolic equations, Dokl. Akad. Nauk SSSR 155 (1964), 285-288=Soviet Math. Dokl. 5 (1964), 409-413. MR 29 \#1439.

Department of Mathematics, University of Auckland, Private Bag, Auckland, New ZeAland 